\title{
Variational Rashba splitting in two-dimensional electron gases in III-V semiconductor heterojunctions
}

\author{
M. A. Toloza Sandoval, ${ }^{1}$ A. Ferreira da Silva, ${ }^{1}$ E. A. de Andrada e Silva, ${ }^{2}$ and G. C. La Rocca ${ }^{3}$ \\ ${ }^{1}$ Instituto de Física, Universidade Federal da Bahia, 40210-340 Salvador, Bahia, Brazil \\ ${ }^{2}$ Instituto Nacional de Pesquisas Espaciais, CP 515, 12201-970 São José dos Campos, SP, Brazil \\ ${ }^{3}$ Scuola Normale Superiore and CNISM, Piazza dei Cavalieri 7, I-56126 Pisa, Italy
}

(Received 13 March 2009; revised manuscript received 27 April 2009; published 3 June 2009)

\begin{abstract}
Control of the Rashba spin-orbit coupling in semiconductor two-dimensional electron gases (2DEGs) is of fundamental interest to the rapidly evolving semiconductor spintronics and depends on the detailed knowledge of the controversial interface and barrier penetration effects. Based on the $8 \times 8 \mathbf{k} \cdot \mathbf{p}$ Kane model for the bulk, we propose a spin-dependent variational solution for the conduction subbands of III-V heterojuctions, which reveals analytically the different contributions to the Rashba splitting and its dependency on heterostructure and band parameters as the band offset and effective masses. Perturbation expansions are used to derive renormalized parameters for an effective, simple, and yet accurate one band model. Spin-dependent modified Fang-Howard trial functions, which satisfy the spin-dependent boundary conditions, are then introduced. The subband splitting is given as a function of the variational parameter which is obtained minimizing the total energy of the 2DEG. Our calculations applied to InAlAs/InGaAs heterojunctions, where a near $20 \%$ increase in the splitting is observed due to the barrier penetration, are in good agreement with both experiment and exact numerical calculations. Well-known expressions in the limit of a perfect insulating barrier are exactly reproduced.
\end{abstract}

DOI: 10.1103/PhysRevB.79.241305

PACS number(s): 73.20.-r, 73.21.Fg, 78.67.De

The desired control of the spin-orbit splitting for twodimensional (2D) electron gases (2DEGs) in III-V semiconductor heterojunctions, as in the Datta and Das spin transistor, has not been achieved yet. The quantitative agreement between theory and experiment is far from complete. Among different studies, there are in particular long-standing controversies concerning the barrier and boundary effects, ${ }^{1,2}$ as well as regarding the splitting dependence on the electron density and the consistency among the different experimental methods. ${ }^{3-5}$

In view of the spintronics, semiconductor heterojunctions form a special class of Rashba split 2DEGs. The electrons are confined by a triangular potential and the strength of the Rashba coupling as well as the electron density $\left(n_{s}\right)$ can be varied with the gate voltage. Different experiments have been quantitatively interpreted with a simple model for the 2DEGs, ${ }^{6}$ i.e., $H_{c}=\hbar^{2}\left(k_{x}^{2}+k_{y}^{2}\right) / 2 m^{*}+\alpha^{*} \boldsymbol{\sigma} \cdot \mathbf{k} \times \mathbf{e}_{z}$, where the Rashba coupling parameter derived from Kane model in the infinite barrier approximation ${ }^{7}$ is given by

$$
\alpha^{*}=\frac{\hbar^{2}}{2 m^{*}} \frac{\Delta}{E_{g}} \frac{2 E_{g}+\Delta}{\left(E_{g}+\Delta\right)\left(3 E_{g}+2 \Delta\right)} e E .
$$

Here the band parameters are those of the well material and $E$ is the confining electric field seen by the 2DEG near the interface. The spin splitting at the Fermi level is then given by $\delta \varepsilon=2 \alpha^{*} k_{F}$. However, this model has some limitations because it does not include nonparabolicity, barrier penetration, and spin-dependent boundary conditions known to lead to sizable corrections. ${ }^{2,8-13}$ There is, however, no simple or consensus way to include or calculate these effects which are usually included through numerical integration of multiband models. ${ }^{2,8-12}$ The problems with such numerical calculations are the spurious solutions, ad hoc operator symmetrization, and the lack of transparency concerning the different contributions to the Rashba splitting and its dependency on the various parameters.

Here, we propose a variational solution for the spinresolved heterojunction electronic structure within the multiband envelope-function approximation which is free from the above concerns, allowing for the analytical discrimination of the different contributions to the strength of the Rashba effect. The dispersion relation of the spin-split conduction subbands is analytically obtained as a function of the variational parameter determined by the exact minimization of the total energy of the 2DEG. The obtained Rashba spinorbit splitting for InAlAs/InGaAs heterojunctions results in reasonably good agreement with recent measurements ${ }^{14}$ and numerical calculations. ${ }^{10-12}$

With the proper choice of parallel wave vector and spinquantization directions, and using the eight-band $\mathbf{k} \cdot \mathbf{p}$ Kane model, the spin-resolved effective Hamiltonian for the 2D conduction subbands can be written as ${ }^{13,15}$

$$
\begin{aligned}
H_{\mathrm{eff} \pm}= & -\frac{\hbar^{2}}{2} \frac{d}{d z} \frac{1}{m\left(z, \varepsilon_{ \pm}\right)} \frac{d}{d z}+\frac{\hbar^{2} k^{2}}{2 m\left(z, \varepsilon_{ \pm}\right)}+E_{c}(z) \\
& +v(z) \mp\left[\frac{d}{d z} \beta\left(z, \varepsilon_{ \pm}\right)\right] k,
\end{aligned}
$$

where $v(z)$ is the confining electrostatic potential, $E_{c}(z)$ is the conduction-band-edge profile, and $m\left(z, \varepsilon_{ \pm}\right)$and $\beta\left(z, \varepsilon_{ \pm}\right)$are described by simple expressions in terms of the bulk band parameters. ${ }^{13} \varepsilon_{ \pm} \equiv \varepsilon_{ \pm}(k)$ are the subband dispersion relations, we are looking for, $k$ being the wave vector parallel to the interface. We indicate with $\Psi_{ \pm}$the conduction-band envelope function for spin up (+) or down (-) along the inplane direction perpendicular to $\mathbf{k}$. From the Schröedinger 


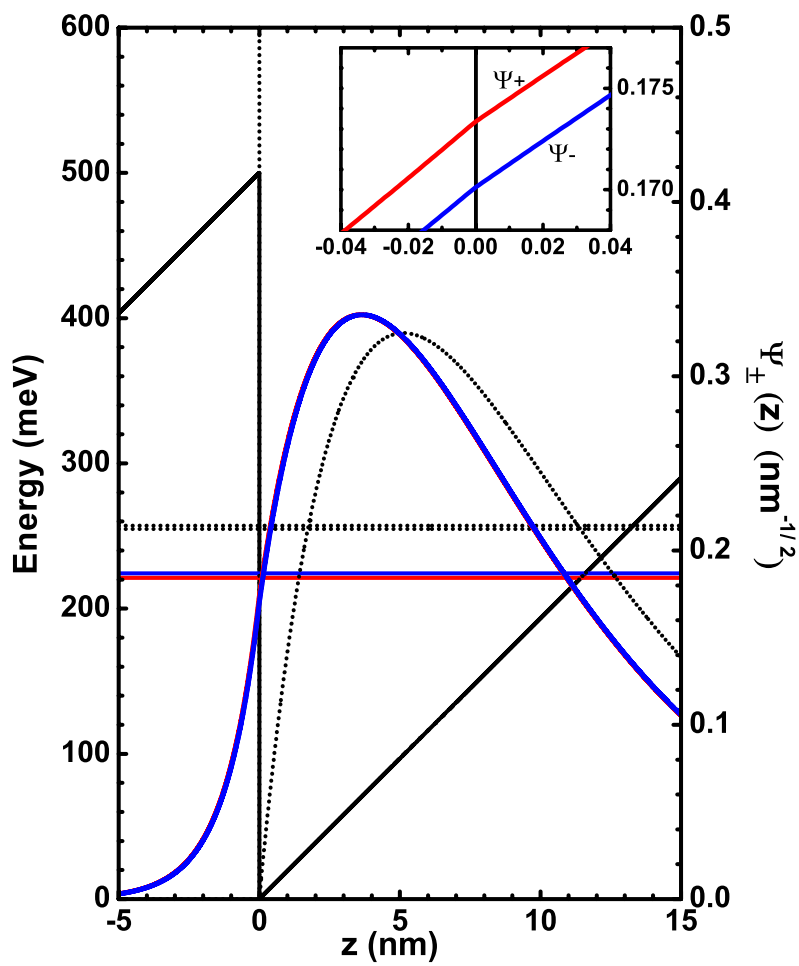

FIG. 1. (Color online) Potential profile obtained envelope functions and spin-split energies at $k_{F}$ for $\operatorname{In}_{0.52} \mathrm{Al}_{0.48} \mathrm{As} / \mathrm{In}_{0.53} \mathrm{Ga}_{0.47} \mathrm{As}$ heterojunctions with $n_{s}=1.4 \times 10^{12} \mathrm{~cm}^{-2}$. Spin-dependent modified Fang-Howard trial functions are shown, with the axis on the right. The dotted lines give the infinite or perfect insulating barrier approximation, i.e., insulator/ $\mathrm{In}_{0.53} \mathrm{Ga}_{0.47}$ As. The inset expands the interface region to show more clearly the spin dependency of the envelope function. The band parameters used are $m^{*}=0.041 m_{e}$, $E_{g}=0.813 \mathrm{eV}$, and $\Delta=0.326 \mathrm{eV}$ for $\mathrm{In}_{0.53} \mathrm{Ga}_{0.47} \mathrm{As}$ and for $\operatorname{In}_{0.52} \mathrm{Al}_{0.48} \mathrm{As}: E_{g}=1.513 \mathrm{eV}, \quad \Delta=0.309 \mathrm{eV} \quad$ and $m^{*}=0.073 m_{e}$ (obtained with the assumption of equal momentum matrix element, fixed with $m^{*}$ in the well). $\varepsilon_{s c}=13.1 \varepsilon_{0}$ and for the conduction-band offset we have used $v_{0}=0.5 \mathrm{eV}$. With these parameters, $\bar{m}_{1}=0.05 m_{e}$.

equation with the above effective Hamiltonian, within standard envelope-function approximation, ${ }^{16}$ one gets that besides $\Psi_{ \pm}$(Refs. 13, 17, and 18)

$$
-\frac{\hbar^{2}}{2 m} \frac{d}{d z} \Psi_{ \pm} \mp \beta k \Psi_{ \pm}
$$

must also be continuous, representing the so-called spindependent boundary conditions for the envelope functions. Considering III-V semiconductor heterojunctions, the 2DEG is confined near the interface by a triangular potential formed by the electrostatic potential plus the conduction-band offset, as illustrated in Fig. 1. In this case, $v(z)=e E z$, with the electric field given by $E=e n_{s} / \varepsilon_{s c}$, where $n_{s}$ is the 2D density of electrons and $\varepsilon_{s c}$ is the dielectric constant. The system is assumed to be infinite along the plane of the interface and differences in $\varepsilon_{s c}$ are neglected for simplicity.

It is shown that by making slightly different approximations for $H_{\text {eff } \pm}$ on the two sides of the heterojunction, it is possible to obtain accurate variational solutions for $\varepsilon_{ \pm}(k)$. In the barrier region $(z<0)$, since our energies of interest lays here inside the gap, we extend the work of Brozak et al. ${ }^{19}$ for the perturbation expansion of the parameters by using a $8 \times 8 \mathbf{~ k} \cdot \mathbf{p}$ model, instead of the $4 \times 4$ model used by them. In this way, we obtain not only the renormalized effective mass $\bar{m}$ but also a renormalized Rashba coupling parameter $\bar{\alpha}$, which include nonparabolicity corrections. In the quantum well region $(z>0)$, following our earlier work references, ${ }^{7,13}$ we expand $m$ and $\beta$ in a power series in terms of the small parameter $\left(\varepsilon_{ \pm}-e E z\right) /\left(E_{g}+\Delta\right)$ and take only the leadingorder terms. As a result, the following effective Hamiltonian is obtained:

$$
H_{\mathrm{eff} \pm}=\bar{H}_{ \pm} \theta(-z)+H_{ \pm}^{*} \theta(z)
$$

with

$$
\bar{H}_{ \pm}=\frac{\hbar^{2}}{2 \bar{m}}\left(-\frac{d^{2}}{d z^{2}}+k^{2}\right)+v_{0}+e E z \mp \bar{\alpha} k
$$

and

$$
\begin{aligned}
H_{ \pm}^{*}= & \frac{\hbar^{2}}{2 m^{*}}\left(-\frac{d^{2}}{d z^{2}}+k^{2}\right)-a \frac{\left[\frac{\hbar^{2}}{2 m^{*}}\left(-\frac{d^{2}}{d z^{2}}+k^{2}\right)\right]^{2}}{E_{g}+\Delta} \\
& +e E z \mp \alpha^{*} k .
\end{aligned}
$$

Note that the band parameters (i.e., $m^{*}, E_{g}$, and $\Delta$ ) to be used in $\bar{H}_{ \pm}$are those of the barrier material, while in $H_{+}^{*}$, those of the well material, and that $v_{0}=E_{c}^{(1)}-E_{c}^{(2)}$ gives the band offset or barrier height. The conduction-band-edge effective mass $\left(m^{*}\right)$, the nonparabolicity parameter $(a)$, and the Rashba coupling $\left(\alpha^{*}\right)$ are exactly as obtained in Ref. 7, while the renormalized parameters in the barrier region read as

$$
\frac{1}{\bar{m}}=\frac{1}{m^{*}} \frac{E_{g}\left(E_{g}+\Delta\right)}{3 E_{g}+2 \Delta} \frac{3\left(E_{g}-v_{0}\right)+2 \Delta}{\left(E_{g}-v_{0}\right)\left(E_{g}-v_{0}+\Delta\right)}
$$

and

$$
\bar{\alpha}=\frac{\hbar^{2}}{2 m^{*}} \frac{E_{g}\left(E_{g}+\Delta\right)}{3 E_{g}+2 \Delta} \frac{2 \Delta\left(E_{g}-v_{0}\right)+\Delta^{2}}{\left(E_{g}-v_{0}\right)^{2}\left(E_{g}-v_{0}+\Delta\right)^{2}} e E .
$$

As expected, they reduce exactly to their corresponding band-edge values $m^{*}$ and $\alpha^{*}$, as $v_{0}$ goes to zero. Accordingly, the boundary conditions are now given by

$$
-\frac{\hbar^{2}}{2 \bar{m}_{1}} \frac{d}{d z} \Psi_{1 \pm} \mp \bar{\beta}_{1} k \Psi_{1 \pm}=-\frac{\hbar^{2}}{2 m_{2}^{*}} \frac{d}{d z} \Psi_{2 \pm} \mp \beta_{2}^{*} k \Psi_{2 \pm},
$$

with

$$
\bar{\beta}=\frac{\hbar^{2}}{2 m^{*}} \frac{\Delta}{3 E_{g}+2 \Delta} \frac{E_{g}\left(E_{g}+\Delta\right)}{\left(E_{g}-v_{0}\right)\left(E_{g}-v_{0}+\Delta\right)}
$$

[note that similarly to $m$ and $\alpha, \beta^{*}=\bar{\beta}\left(v_{0}=0\right)$ ]. Hereafter we shall use for all the parameters index 1 in the barrier region and index 2 in the quantum well region.

We now introduce the following spin-dependent modified Fang-Howard trial functions that satisfy the above conditions and allow a simple variational solution for the first subband, 
TABLE I. The different terms of the effective Hamiltonian used in the calculation of the energy expectation values and the Rashba variational splitting.

\begin{tabular}{lc}
\hline \hline$T_{ \pm}$ & $\left\langle\hbar^{2} / 2\left[1 / \bar{m} \theta(-z)+1 / m^{*} \theta(z)\right]\left(-d^{2} / d z^{2}+k^{2}\right)\right\rangle_{ \pm}$ \\
$T_{n p \pm}$ & $\left\langle-a\left(\left[\hbar^{2} / 2 m^{*}\left(-d^{2} / d z^{2}+k^{2}\right)\right]^{2} / E_{g}+\Delta\right) \theta(z)\right\rangle_{ \pm}$ \\
$V_{ \pm}$ & $\left\langle e E z+v_{0} \theta(-z)\right\rangle_{ \pm}$ \\
$V_{\text {so } \pm}$ & $\left\langle\mp\left[\bar{\alpha} \theta(-z)+\alpha^{*} \theta(z)\right] k\right\rangle_{ \pm}$ \\
\hline
\end{tabular}

including the Rashba, nonparabolicity, and barrier penetration effects,

$$
\Psi_{ \pm}(z)=\left\{\begin{array}{cc}
\Psi_{1 \pm}=A_{ \pm} e^{k_{b} z / 2}, & z \leq 0 \\
\Psi_{2 \pm}=B_{ \pm}\left(z+c_{ \pm}\right) e^{-b z / 2}, & z \geq 0,
\end{array}\right.
$$

where $k_{b}=2 \sqrt{2 \bar{m}_{1} v_{0} / \hbar^{2}}$ and $b$ is the variational parameter. ${ }^{20}$ The boundary conditions impose $A_{ \pm}=B_{ \pm} c_{ \pm}$and lead to

$$
c_{ \pm}=\frac{2}{b+\frac{m_{2}^{*}}{\bar{m}_{1}} k_{b} \pm 4 \frac{m_{2}^{*}}{\hbar^{2}}\left(\bar{\beta}_{1}-\beta_{2}^{*}\right) k},
$$

where it is seen an analytical and explicit coupling between the spin and the dynamic variable $k$ of the 2D electrons, which can be of great interest in the study of spin photocurrents, for example. Finally, normalization sets $B_{+}=\sqrt{\left(b^{3} / 2\right) /\left[1+b c_{+}+b^{2} c_{+}^{2} / 2\left(1+b / k_{b}\right)\right]}$.

Using these trial functions for the spin-polarized first subbands, the energy levels will be given by

$$
\varepsilon_{ \pm}(k)=\left\langle\Psi_{ \pm}\left|H_{\mathrm{eff} \pm}\right| \Psi_{ \pm}\right\rangle=\left\langle H_{\mathrm{eff} \pm}\right\rangle_{ \pm},
$$

where, for a given electron density $n_{s}$, the value of $b$ is determined by minimizing the total energy, i.e., the sum over all the electrons, considering the electron-electron interaction in the Hartree approximation. For the heterojunction in Fig. 1 , we obtain $b=0.42 \mathrm{~nm}^{-1}$ ( $b=0.39 \mathrm{~nm}^{-1}$ for insulator/ InGaAs); the dependence with both $k$ and spin is negligible. The expectation values $\left\langle H_{\text {eff }}\right\rangle_{ \pm}$composed by the different terms listed in Table I are easily calculated ${ }^{21}$ and in the limit of infinite barrier reduce exactly to the previous results in Ref. 7.

Figures 1 and 2 show the obtained results for a 2DEG in an $\mathrm{In}_{0.52} \mathrm{Al}_{0.48} \mathrm{As} / \mathrm{In}_{0.53} \mathrm{Ga}_{0.47} \mathrm{As}$ heterojunction with $n_{s}=1.4 \times 10^{12} \mathrm{~cm}^{-2}$. In Fig. 1 we show the energy levels at $k_{F}$ and the modified Fang-Howard envelope function in both finite and infinite barrier (dotted line) approximations. The spin dependence can be seen in the inset with a closer view of the interface region. Figure 2(a) shows the spin splitting as a function of the parallel wave vector. In the inset, we show the subband dispersion relations, with the parabolic approximation (dashed line). The splitting up to $k_{F}$ is seen to be linear with $k$; but for larger $k s$, it flattens and reaches a maximum as expected. ${ }^{10,13}$ From the obtained energy splitting of the states at the Fermi level, i.e., $\delta \varepsilon=\left|\left\langle H_{\text {eff }}\right\rangle_{+}-\left\langle H_{\text {eff }-}\right\rangle_{-}\right|=2 \alpha_{\text {eff }} k_{F}$, an effective (variational) Rashba coupling parameter $\alpha_{\text {eff }}$ can be defined, which in this case is seen to be around 20\% larger than $\alpha^{*}$ in Eq. (1), corresponding to the infinite barrier approximation. This is
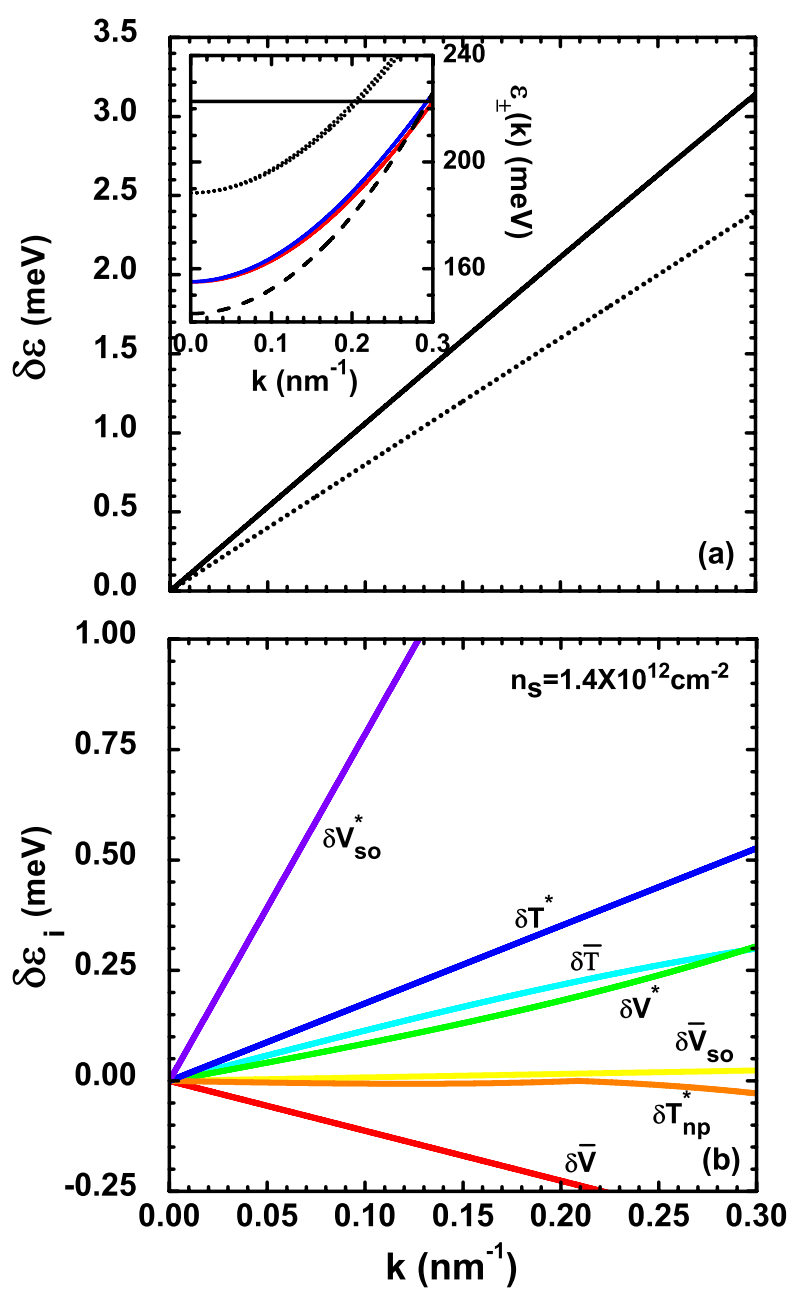

FIG. 2. (Color online) (a) Total Rashba spin-orbit splitting for a $\mathrm{In}_{0.52} \mathrm{Al}_{0.48} \mathrm{As} / \mathrm{In}_{0.53} \mathrm{Ga}_{0.47} \mathrm{As}$ heterojunction. The inset shows the spin-split conduction subband dispersion relations and parabolic approximation (dashed line). The dotted lines show the limit case, i.e., insulator $/ \mathrm{In}_{0.53} \mathrm{Ga}_{0.47} \mathrm{As}$. (b) Rashba spin-splitting contributions.

due to different new contributions allowed with barrier penetration. In the lower panel [Fig. 2(b)], these different contributions are plotted with the notation of Table I. The effective Hamiltonian (4) and the corresponding Rashba splitting can be written as a sum of the different terms listed in Table I, i.e., $H_{\mathrm{eff} \pm}=\sum_{i} H_{\mathrm{eff} \pm}^{(i)}$, and $\delta \varepsilon=\sum_{i} \delta \varepsilon_{i} \geq 0$, respectively, with $\delta \varepsilon_{i}=\left\langle H_{\mathrm{eff}-}^{(i)}\right\rangle_{-}-\left\langle H_{\mathrm{eff}}^{(i)}\right\rangle_{+}$. These contributions can be further separated into barrier and well components, recalling that $T_{ \pm}=\bar{T}_{ \pm}+T_{ \pm}^{*}$ and similarly for $V$ and $V_{\mathrm{so}}$.

It is interesting to note that all the contributions are not exactly but approximately linear with $k$ and that there are both positive and negative new contributions due to barrier penetration. In the limit of infinite barrier, only $\delta V_{\mathrm{so}}^{*}$ survives, all the other terms go to zero (the envelope function becomes independent of the spin and no contributions from the kinetic and potential energies in the well are seen), and one gets $\alpha_{\text {eff }}=\alpha^{*}$. Instead, when barrier penetration is allowed due to the spin-dependent trial functions, all the different terms in $H_{\text {eff }}$ have different expectation values for each spin direction. As seen in Fig. 2(b), they partially cancel each other, leading anyway to a net increase in the total splitting. 
The biggest contribution is from the kinetic energy (within both well and barrier) due to the small difference between the envelope functions with opposite spins shown in Fig. 1.

Recently, Yang et al. ${ }^{14}$ studied the Shubnikov-de Haas oscillations in an $\operatorname{In}_{0.52} \mathrm{Al}_{0.48} \mathrm{As} / \operatorname{In}_{x} \mathrm{Ga}_{1-x}$ As heterojunction with $x=0.53$ near the interface and $n_{s}=1.4 \times 10^{12} \mathrm{~cm}^{-2}$ and measured a difference in the spin-resolved population corresponding to $\delta n / n_{s} \sim 0.023 \quad\left(\delta n=\left|n_{+}-n_{-}\right|\right)$. Our model gives $\delta n / n_{s} \sim 0.026 \quad\left(n_{ \pm}=k_{ \pm}^{2} / 4 \pi\right)$ corresponding to $\alpha_{\text {eff }}=5.2 \times 10^{-12} \mathrm{eV} \mathrm{m}$, which is reduced to $\alpha^{*}=4.0 \times 10^{-12} \mathrm{eV} \mathrm{m}$ in the infinite barrier approximation. This indicates that Rashba is the dominant spin-orbit term in this structure. In fact using an effective Dresselhaus parameter $\gamma \sim 30 \mathrm{eV} \AA^{3}, 22$ we estimate a pure Dresselhaus splitting $\delta \varepsilon_{D} \sim 2 \gamma k_{F}\left(b^{2} / 4\right) \sim 0.7 \mathrm{meV}$, three times smaller than the obtained Rashba splitting. ${ }^{23}$ Besides such correction, the detailed comparison with experiment is not possible due to the uncertainty in the actual confining electric field.

To conclude, we have presented a variational framework for the calculation of the Rashba spin-orbit splitting in III-V semiconductor heterojunctions, which leads to accurate and simple expressions, with explicit dependence on the different bulk band parameters and reveals the different contributions to the Rashba splitting. It has been applied here to InGaAs inversion layers; nevertheless the theory is general and can be applied to any III-V heterojunction. Preliminary results for GaAs and InSb heterojunctions show also similar good agreement with previous studies. ${ }^{21}$ The transparency and simplicity of the present model can be of great help to fabricate and/or optimize new devices in spintronics. It also helps clarifying the controversial role of the interface in the Rashba effect. Finally, good agreement with both experimental data and exact numerical calculations further supports its accuracy.

The authors thank the Brazilian agencies CNPq, CAPES, and FAPESB for financial support.
${ }^{1}$ I. Zutic, J. Fabian, and S. Das Sarma, Rev. Mod. Phys. 76, 323 (2004).

${ }^{2}$ U. Ekenberg and D. M. Gvozdic, Phys. Rev. B 78, 205317 (2008).

${ }^{3}$ T. Matsuyama, R. Kürsten, C. Meißner, and U. Merkt, Phys. Rev. B 61, 15588 (2000).

${ }^{4}$ C. Schierholz, T. Matsuyama, U. Merkt, and G. Meier, Phys. Rev. B 70, 233311 (2004).

${ }^{5}$ M. Akabori, V. A. Guzenko, T. Sato, T. Schapers, T. Suzuki, and S. Yamada, Phys. Rev. B 77, 205320 (2008).

${ }^{6}$ E. I. Rashba, Sov. Phys. Solid State 2, 1109 (1960); Yu. A. Bychkov and E. I. Rashba, JETP Lett. 39, 78 (1984).

${ }^{7}$ E. A. de Andrada e Silva, G. C. La Rocca, and F. Bassani, Phys. Rev. B 50, 8523 (1994).

${ }^{8}$ Jun Li, Kai Chang, G. Q. Hai, and K. S. Chan, Appl. Phys. Lett. 92, 152107 (2008).

${ }^{9}$ S. Lamari, Phys. Rev. B 75, 155302 (2007).

${ }^{10}$ W. Yang and K. Chang, Phys. Rev. B 73, 113303 (2006).

${ }^{11}$ W. Zawadski and P. Pfeffer, Semicond. Sci. Technol. 19, R1 (2004).

${ }^{12} \mathrm{R}$. Winkler, Spin-Orbit Coupling Effects in Two-Dimensional Electron and Hole Systems, Springer Tracts in Modern Physics Vol. 191 (Springer-Verlag, Berlin, 2003).

${ }^{13}$ E. A. de Andrada e Silva, G. C. La Rocca, and F. Bassani, Phys.
Rev. B 55, 16293 (1997).

${ }^{14}$ C. L. Yang, H. T. He, L. Ding, L. J. Cui, Y. P. Zeng, J. N. Wang, and W. K. Ge, Phys. Rev. Lett. 96, 186605 (2006).

${ }^{15}$ L. G. Gerchikov and A. V. Subashiev, Sov. Phys. Semicond. 26, 73 (1992).

${ }^{16}$ M. G. Burt, J. Phys.: Condens. Matter 4, 6651 (1992).

${ }^{17}$ F. T. Vasko, JETP Lett. 30, 541 (1979).

${ }^{18}$ R. A. Suris and A. B. Sokolski, Sov. Phys. Semicond. 21, 529 (1987).

${ }^{19}$ G. Brozak, E. A. de Andrada e Silva, L. J. Sham, F. DeRosa, P. Miceli, S. A. Schwarz, J. P. Harbison, L. T. Florez, and S. J. Allen, Phys. Rev. Lett. 64, 471 (1990).

${ }^{20}$ G. Bastard, Wave Mechanics Applied to Semiconductor Heterostructures (Les Editions de Physique, Les Ulis, 1990).

${ }^{21}$ M. A. Toloza Sandoval, A. Ferreira da Silva, E. A. de Andrada e Silva, and G. C. La Rocca (unpublished).

${ }^{22}$ J. M. Jancu, R. Scholz, E. A. de Andrada e Silva, and G. C. La Rocca, Phys. Rev. B 72, 193201 (2005).

${ }^{23}$ Recent data in Ref. 5 indicate that in InAlSb/InGaSb 2DEGs instead, the Dresselhaus contribution can dominate. However, a different extended trial function is necessary when discussing this common case of narrow wells with barrier penetration on both sides. 